INTERNATIONAL DESIGN CONFERENCE - DESIGN 2018

https://doi.org/10.21278/idc.2018.0133

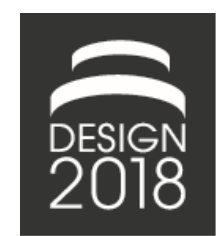

\title{
MEASUREMENT OF BRAIN ACTIVITIES OF IDEA GENERATION (SKETCH)
}

\author{
T. Kato, H. Okada and Y. Izu
}

\begin{abstract}
This study analyzed the correspondence between the brain activities and some types of the idea generation by sketching. To compare the types on the basis of the brain activities, this study employed a NIRS apparatus which measures the change in concentration of oxygenated hemoglobin in cerebral blood flow and detects the activated areas in the prefrontal cortex. The result suggests the idea generation with the sketch based on long term memory activates the prefrontal cortex more than that based on a previous sketch.
\end{abstract}

Keywords: idea generation, sketching, NIRS, design thinking

\section{Introduction}

The mission of design should be the improvement of not only material (physical) values but also spiritual values (Matsuoka, 2000). Recently, the objective of the product development has shifted from the multifunctionality and high performance to the fulfilment of user's spiritual values and creating the experiences of users. To achieve the goal, the problem solving activities and the thinking process of designers are focused in various fields, and the tool development and the education regarding design are carried out in various places (Plattner et al., 2011). Their effectiveness, however, is evaluated subjectively and is difficult to be confirmed because of the fluctuation by the personal difference. This causes the need for an objective evaluation method.

The research measuring the brain activities against the design tasks has become popular due to the development of the measurement devices. Alexiou et al. (2011) compared the brain activities between the ill- and well-structured design problems (furniture layout problems) using a function Magnetic Resonance Imaging (fMRI) apparatus and suggested that the former problem whose evaluation criteria is not well specified activates more brain regions than the latter one. Particularly, the former task activated the two types of the brain regions: one includes the areas involved in visual imagery, semantic processing, and multi-sensory integration, such as the temporal, occipital, and parietal regions; the other is the prefrontal cortex (PFC) for constructing executive schemes of action. Kowatari et al. (2009) measured the brain activities of the undergraduate/graduate students designing the shape of a pen using a Magnetic Resonance Imaging (MRI) device and concluded the design task facilitated and suppressed the right and left PFCs, respectively. Folley and Park (2005) employed a Near Infrared Spectroscopy (NIRS) apparatus and compared the brain activities between schizophrenic patients and healthy participants during the task to generate new uses of the objects shown in the computer screen. As a result, compared to the healthy participants, the patients generated more uses, and the more brain regions in both the right and left PFCs were activated. Gibson et al. (2009) also compared the brain activities between the creative individuals (musicians) and healthy participants. The result reveals that the musicians also derived more uses and activated more brain regions in the PFCs, same as the patients. 
Nagamori et al. (2009) measured the brain activities of the undergraduate/graduate students when they work on the following two creative tasks using NIRS apparatus. Task 1 is to select and arrange one or three colors which are fit to the given concept (adjective phrase, such as "cool" and "cute"). Task 2 is to make a "cute chair" using single- or multi-color blocks. The results of the two tasks show some task conditions (e.g., selecting one color in Task 1 and using multi-color blocks in Task 2) activate the PFC more than the others. In other words, the more creativity the task requires, the more brain regions in the PFC activate. Kato et al. (2016) compared the brain activities of the participants when they conducted the Finke's pattern generation (form assemble) task (Finke et al., 1992) employing the hand drawing and computer operation. The result shows the latter one activates the right PFC more, and the activation seems to be occurred by the ways of idea transformations (not occurred by the assembling methods: hand drawing and computer operation).

These studies confirmed the design tasks activate the brain regions in the PFC, suggesting the possibilities to evaluate how the person is creative state during the design task by measuring them.

The present study is a preliminary stage to clarify the relationship between the design activity and its brain activities and aims to correlate the brain activities with the design using sketches which is one of the common tools in design activities. This paper is organized as follows. Section 2 illustrates the types of the idea generations by sketching. Section 3 presents the method to measure the brain activities. Section 4 describes the result and discussion of the brain activity measurement experiment, while Section 5 provides conclusions and future tasks.

\section{Classification of sketch (drawing) generation}

This study focuses on the classification of the sketch (drawing) generation proposed by Goel (1995). This classification includes two types of drawing generations as follows. One is "new generation (NG)" that generates a drawing using the long term memory (LTM) of the person. LTM stores the information regarding what the parson has encountered and is used to make logical deductions, to understand ideas, as well as to memorize fact. The other is "transformation" which generates a drawing based on the previously generated drawing. The transformation is further classified into two types: "lateral transformation (LT)" that modifies a drawing into another related, but distinctly different, drawing; "vertical transformation (VT)" which reiterates and reinforces an existing drawing through explication and detailing. The three types of the drawing generations are illustrated using some sketch examples in Figure 1. These sketches were drawn for the idea generation of USB flash memory during the experiment described in the latter section. Figure 1(a)-(f) display the sketches in order from the sketch generated earlier. Sketch (a) was drawn firstly (i.e. drawn without referring any sketch) and is categorized as NG. Sketch (b) was drawn secondly but drawn without referring Sketch (a) and is also categorized as NG. Sketch (c) is the detail (i.e. the parts action) of Sketch (b) and is categorized as VT. Sketch (d) is categorized as VT because it was generated by referring the side-surface shape of Sketch (c). Sketch (e) is categorized as VT because it illustrates the parts action of Sketch (d). Note that the categorization requires the opinion (concept) of the person who draws the sketch. The aforementioned categorization was done using the interview of them.

This study compared the aforementioned idea generation types using the brain activities measured when people conduct the idea generation. The following section illustrates the method to measure the brain activities.

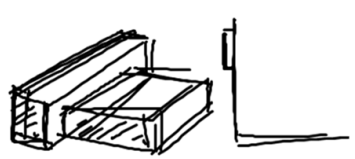

(a) $\mathrm{NG}$

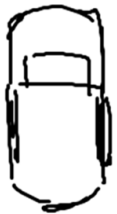

(b) NG

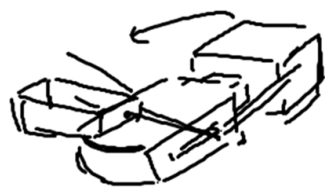

(c) VT

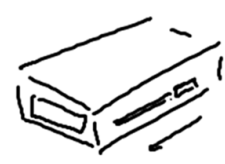

(d) LT

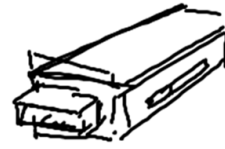

(e) VT

Figure 1. Example of idea generation types 


\section{Methods to measure and analyze brain activities}

\subsection{Brain activities measuring method}

The brain activities measuring methods include NIRS, PET (Positron Emission Tomography), SPECT (Single Photon Emission Computed Tomography), MRI (Magnetic Resonance Imaging), EEG (Electroencephalogram), MEG (Magnetoencephalogram), and so on. This study employed NIRS because of the following reasons: 1) NIRS is a no-invasive measurement and does not constrain the participant's movements (sketching); 2) NIRS apparatus has a high time resolution (less than $1 \mathrm{sec}$ ) which enables to measure the brain activities at the moment of the idea generation by sketching.

A NIRS apparatus is composed of some emitter-detector pairs of near infrared light whose wavelength is from $700 \mathrm{~nm}$ to $1000 \mathrm{~nm}$ and cannot be easily absorbed in a biological tissue. Each emitter has two continuous laser diodes and irradiates the near infrared light of two different wavelength in order to measure the concentration changes in both oxygenated hemoglobin (oxyHb) and deoxygenated hemoglobin (deoxyHb). While the amounts are calculated on the basis of the modified Beer-Lambert Law (Delpy et al., 1988), which gives the relation equation between the attenuation of light and the density changes in light absorber. The measurement principle of NIRS apparatus based on the modified Beer-Lambert Law is described in Figure 2. The equation in this figure shows the concentration changes in oxyHb and deoxyHb $\left(\Delta c_{\text {oxy }}\right.$ and $\left.\Delta c_{\text {deoxy }}\right)$ can be estimated using the logarithm of the ratio between the input and output light intensities ( $I_{\text {in }}$ and $I_{\text {out }}$ ). However, the concentration changes derived from neither different participants nor measurement regions can be compared because the light pass length $\mathrm{d}$ varies according to them. The change in the oxyHb correlates the change in the regional cerebral blood flow. The apparatus, therefore, can capture the change generated by the activation of the nervous activities of the brain.

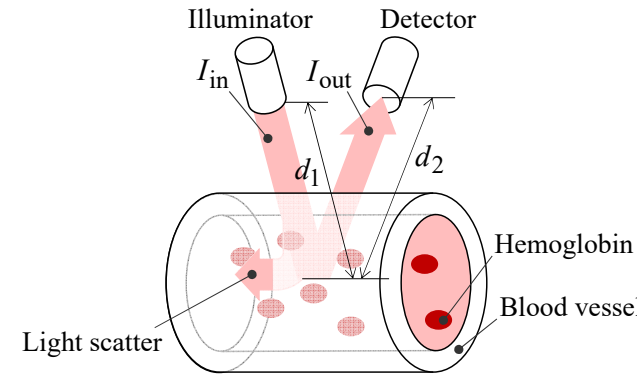

$$
\begin{aligned}
& \log \left(I_{\text {in }} / I_{\text {out }}\right) \cong \varepsilon_{\text {oxy }} \times \Delta c_{\text {oxy }} \times d+\varepsilon_{\text {deoxy }} \times \Delta c_{\text {deoxy }} \times d \\
& \left(\begin{array}{l}
I_{\text {in }}: \text { Input light intensity } \\
I_{\text {out }}: \text { Transmitted intensity } \\
d\left(=d_{1}+d_{2}\right): \text { Light path length } \\
\Delta c_{\text {oxy }}, \Delta c_{\text {deoxy }}: \begin{array}{l}
\text { Density changes in oxy- and } \\
\text { deoxy-hemoglobin }
\end{array} \\
\varepsilon_{\text {oxy }}, \varepsilon_{\text {deoxy }}: \text { Molar absorption coefficients of } \\
\text { oxy- and deoxy-hemoglobin }
\end{array}\right)
\end{aligned}
$$

Figure 2. Measurement principle of NIRS apparatus

This study employed a NIRS device (OEG-16, Spectratech Inc., Tokyo, Japan, Figure 3(a)). This device includes six pairs of laser and photo diodes (illuminators and detectors) whose distance is $30 \mathrm{~mm}$ (Figure 3(b)). The number of the measurement points is sixteen, and they locate between each pair of laser and photo diodes. The measurement brain regions corresponding to the points, termed as channels $(\mathrm{CHs})$, are in the PFC (Figure $3(\mathrm{c})$ ). The sampling frequency is $1.6 \mathrm{~Hz}$.

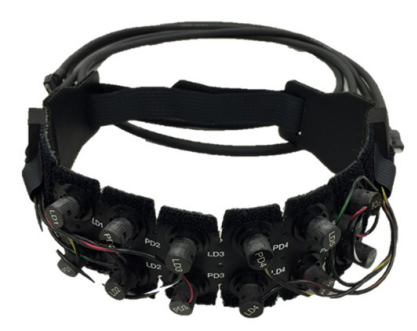

(a) NIRS (OEG-16)

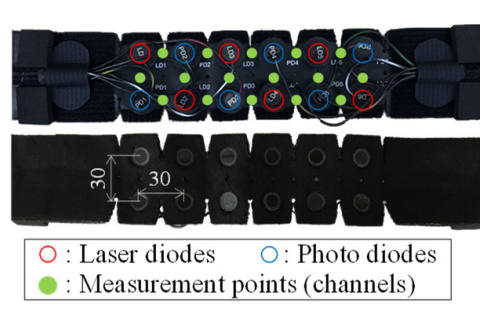

(b) Location of laser and photo diodes

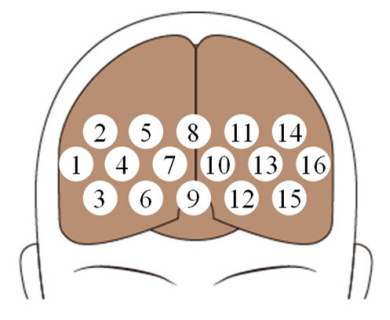

(c) Measured regions termed as channels (chs)

Figure 3. Measurement principle of NIRS apparatus 


\subsection{Analysis method}

This study adopted the change in oxyHb which is the most sensitive indicators of the change in regional cerebral blood flow (Hoshi et al., 2009) and analyzed the signal using the two methods: the moving average processing (Atsumori et al., 2010) and base line correction (Peña et al., 2003). The former removes the components originating from the slow fluctuations of cerebral blood flow, heartbeat, and body motion. In this study, the oxyHb signal was bandpass filtered between 0 and $0.2 \mathrm{~Hz}$ by employing a movement average value in five seconds. The latter removes the component originating from the fatigue of a participant which changes the oxyHb caused linearly with respect to time. In this study, the line connecting the average values of the two control tasks conducted before and after the target task was calculated and was subtracted from the oxyHb signals.

The following section shows the experiment of the brain activity measurement to correlates the three idea generation types and their brain activities.

\section{Brain activity measurement experiment}

\subsection{Measurement procedure of brain activities}

The twelve right-handed healthy designers and graduate/undergraduate students, who specialize in product design, participated in this experiments ( 5 product designers and 7 students specializing product design; 8 male and 4 female). Before each experiment, informed consent was obtained from all of them. The ethics committee of Keio University approved this experiment.

The block design of this experiment is depicted in Figure 4. In the control task 1 and 2, the participants moved their hands and arms as if they sketch something for 30 seconds. This aims to extract the brain activities generated by the idea generation by arranging the participant's movement. Whereas, in the idea generating task, they generated ideas by sketching for 300 seconds on the basis of the five design themes (design objects): scissors, mechanical pencil, USB flash memory, cutter knife, and stapler. Note that the participants were asked only to do sketching and were free to choose the types or methods of sketching. The reason why these everyday things were chosen as design objects is to minimize the difference of the knowledge about them between the participants. Note that they were allowed to generate several sketches within the prescribed time but were prohibited to delete the generated sketches.

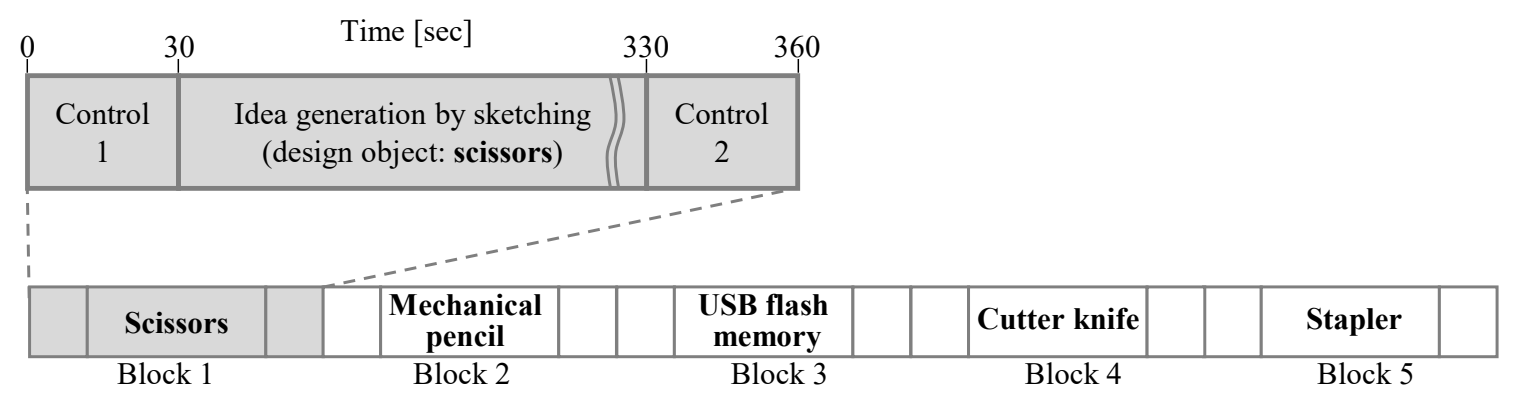

Figure 4. Timing of control and idea generation tasks

This study employed a NIRS apparatus (OEG-16, Spectratech Inc., Tokyo, Japan), described in the preceding section, and an eye tracking system (Talk Eye Lite, Takei scientific instrument Co., Ltd., Niigata, Japan) and measured the cerebral blood flow (the concentration changes in oxyHb) and the points of gaze. Then, the experimenter performed an interview in order to get the information to classify the idea generations in the tasks into the three types described in Section 2. In the interview, the experimenter asked one or two questions described in in Figure 5 for classifying each sketch (idea). Note that the measured points of gaze were displayed to the participants for reminding them of the idea generation during the interview. 


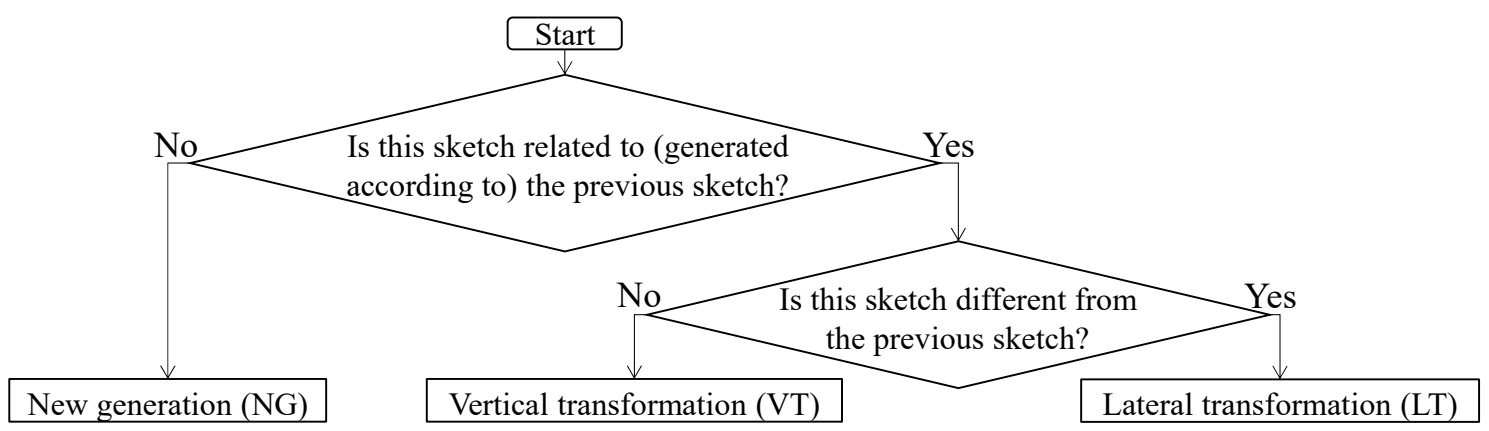

Figure 5. Flowchart to classify idea generation types

The experiment cannot equalize the start time and the implementation time of each sketch generation. Additionally, all the sketch generations cannot be arranged between the control tasks. This means that the cerebral blood flow measurement data (the concentration change in oxyHb $\Delta c_{\text {oxy }}$ ) of a task (sketch generation) is influenced by that of the previous task. This study, therefore, dealt with the data as shown in Figure 6. This figure shows a waveform of cerebral blood flow and time ranges of some sketch generations and control tasks. As shown in this figure, this study calculated the difference between the $\Delta c_{\text {oxy }}$ at the start point of generating the sketch and maximal/minimal $\Delta c_{\text {oxy }}$ during the generation as a brain activation amount and compared them between the sketch generation types. Note that either the maximal or minimal $\Delta c_{\text {oxy }}$ is chosen according to the absolute value of the difference as described in Figure 6.

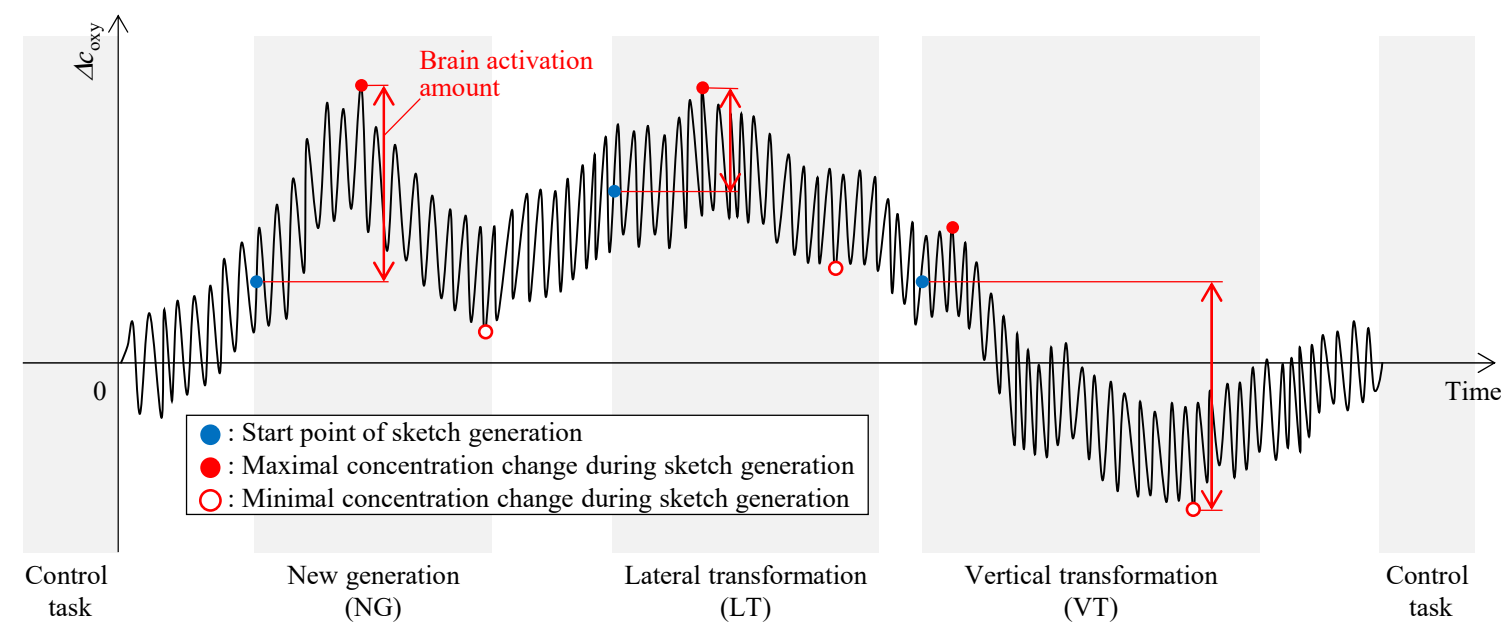

Figure 6. Measurement data of concentration changes in oxygenated hemoglobin (OdyHb)

\subsection{Results and discussions}

The examples of the sketches drawn in the experiment and the number of sketches categorized to each idea generation type are shown in Figure 7 and Table 1, respectively. In this figure, the numbers express the order that the sketches were drawn. Sketch (1) was generated from LTM and based on this, sketches (2) to (5) were drawn. Then, sketch (6) was obtained from LTM and sketch (7) was derived on the basis of sketches (1) and (2). Finally, sketches (8) and (9) were generated in order to explicate sketches (8) and (4), respectively. In Table 1, the asterisks of the participant numbers denote that the participant is a professional designer. Additionally, the red colored figures mean that they are not enough to be used in the statistical tests and were eliminated in this study. Note that, there is no statistical different between the numbers of the designers and students. 


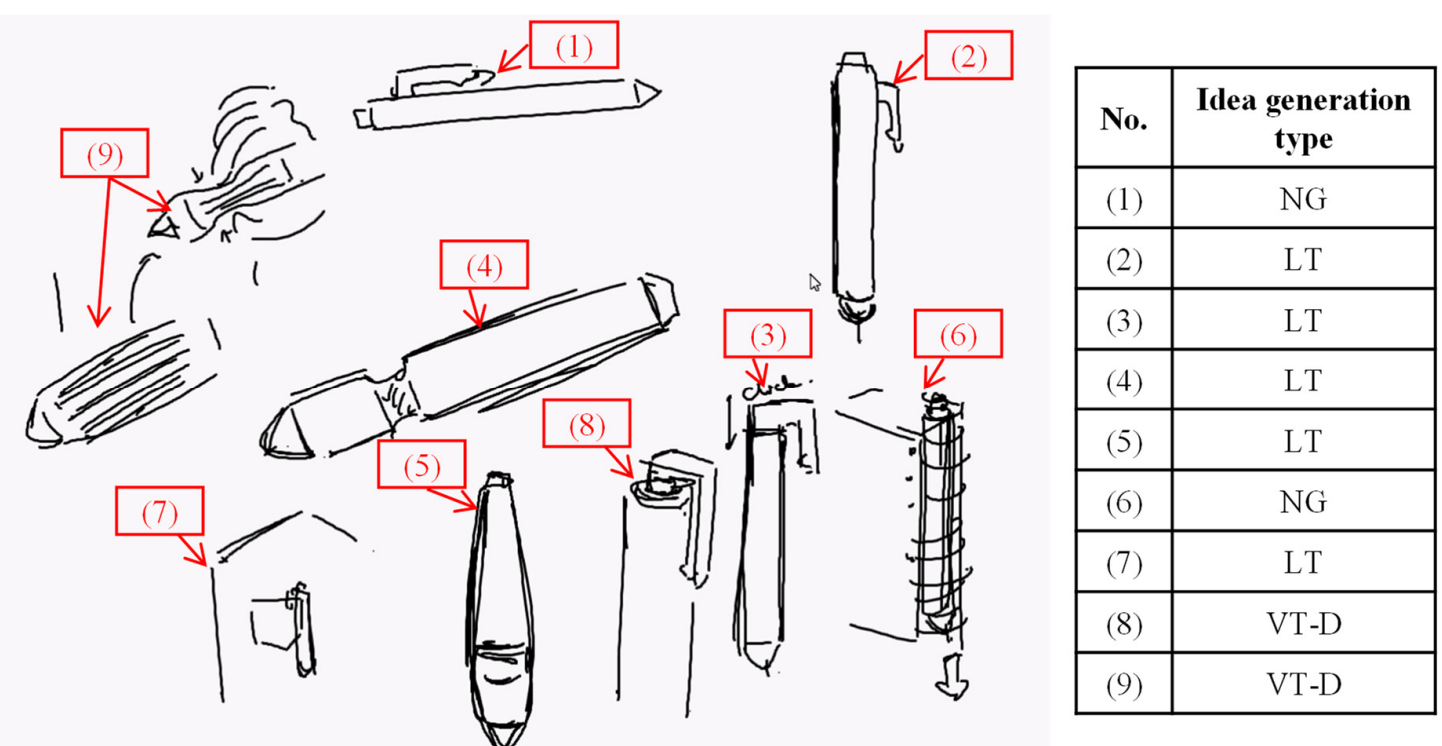

Figure 7. Sketch examples and their idea generation types

Table 1. Number of sketch examples derived by each idea generation type

\begin{tabular}{|c|c|c|c|c|}
\hline $\begin{array}{l}\text { Participant } \\
\text { No. }\end{array}$ & $\begin{array}{l}\text { New generation } \\
\text { (NG) }\end{array}$ & $\begin{array}{l}\text { Lateral transformation } \\
\text { (LT) }\end{array}$ & $\begin{array}{l}\text { Vertical transformation } \\
\text { (VT) }\end{array}$ & Total \\
\hline $1 *($ male $)$ & 7 & 4 & 8 & 19 \\
\hline $2 *$ (male) & 17 & 16 & 16 & 49 \\
\hline $3 *($ male $)$ & 22 & 22 & 27 & 71 \\
\hline $4 *$ (male) & 12 & 14 & 7 & 33 \\
\hline $5^{*}$ (female) & 16 & 0 & 10 & 26 \\
\hline 6 (male) & 20 & 11 & 2 & 33 \\
\hline 7 (female) & 14 & 1 & 10 & 25 \\
\hline 8 (female) & 20 & 7 & 4 & 31 \\
\hline 9 (female) & 19 & 0 & 2 & 21 \\
\hline 10 (male) & 8 & 4 & 2 & 14 \\
\hline 11 (male) & 23 & 6 & 8 & 37 \\
\hline 12 (male) & 10 & 0 & 6 & 16 \\
\hline
\end{tabular}

The Wilcoxon-Mann-Whitney test was performed to compare the brain activation amount data among the three sketch generation types. As a result, the significant difference in the activation amount between NG and LT/VT was confirmed in many participants and brain areas (channels) as shown in Figure 8. This figure shows the results of the Wilcoxson-Mann-Whitney test between the two sketch generation types described on the top of each column. For example, the top left figure means the channel No.6 of the first participant was activated when he did NG task, compared to LT task. This figure suggests the followings. NG (idea generation with the sketch based on LTM) activates the prefrontal cortex (PFC) more than that based on a previous drawing (sketch). The activation of PFC in creative tasks was confirmed in some previous studies (Kowatari et al., 2009; Alexiou et al., 2011). This suggests the brain activity of NG is similar to that of the creative tasks and indicates the possibilities to differentiate the sketch idea generation types on the basis of the creativity. However, the relationship between the types and creativity has not been clearly confirmed and is a future task. Additionally, this study also shows the fluctuation of the activated regions between the participants. Hence, the further investigation is required to achieve the classification of the types. 


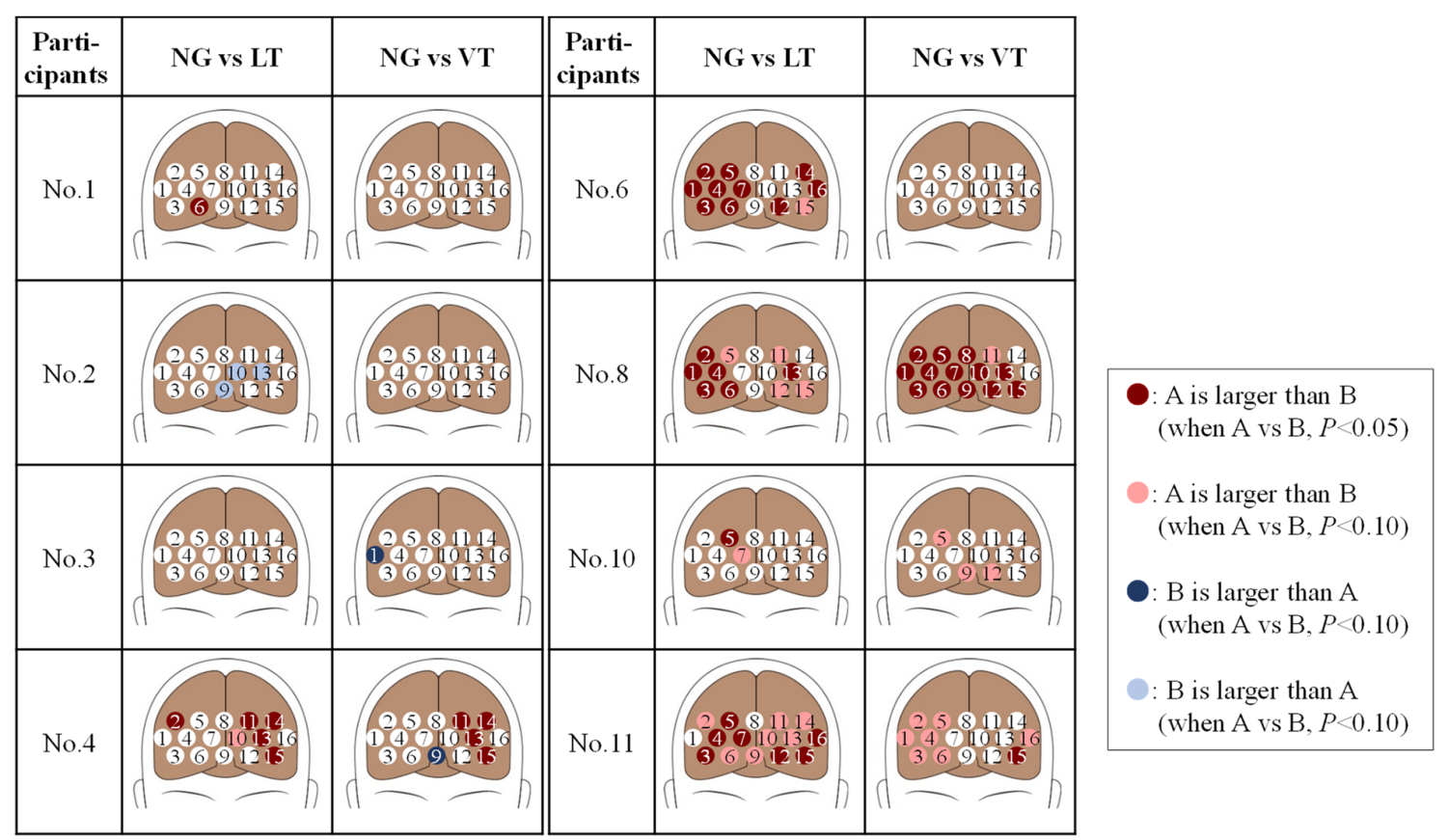

Figure 8. Comparison of activated brain region between idea generation types

\section{Conclusions}

This study employed a NIRS apparatus to measure the brain activities (the concentration change in the oxygenated hemoglobin of the prefrontal cortex) when the participant generates design ideas by sketching. The Wilcoxon-Mann-Whitney test was performed to compare the brain activities (brain activation amounts) among the three types of the sketch generations. The result reveals that new generation, which is one of the idea generation methods defined by Goel and employs the sketch based on long term memory, activates the prefrontal cortex more than that based on a previous drawing (sketch).

The future work is required to increase the number of the participants and indicate the generality of the result.

\section{Acknowledgment}

This work was partly supported by the Japan Society for the Promotion of Science, Grant-in-Aid for Scientific Research (C) (15K00692) and Grant-in-Aid for Young Scientists (B), and was conducted with the permission from the ethics committee of Keio University and the informed consents from all participants. We would like to our gratitude to all of them.

\section{References}

Alexiou, K., Zamenopoulous, T. and Gilbert, S. (2011), "Imaging the designing brain: a neurocognitive exploration of design thinking", Design Computing and Cognition'10, Springer, pp. 489-504. https://doi.org/10.1007/978-94-007-0510-4_26

Atsumori, H., Kiguchi, M., Katura, T., Funane, T., Obata, A. and Sato, H. (2010), "Noninvasive imaging of prefrontal activation during attention-demanding tasks performed while walking using a wearable optical topography system", Journal of Biomedical Optics, Vol. 15 No. 4, pp. 046002. https://doi.org/10.1117/1.3462996

Delpy, D.T., Cope, M., Zee, P., Arridge, S., Wray, S. and Wyatt, J. (1988), "Estimation of optical pathlength through tissue from direct time of flight measurement", Physics in Medicine and Biology, Vol. 33 No. 12, pp. 1433-1442. https://doi.org/10.1088/0031-9155/33/12/008

Finke, R.A., Ward, T.B. and Smith, S.M. (1992), Creative Cognition: Theory, Research, and Applications, MIT Press, Cambridge. 
Folley, B.S. and Park, S. (2005), "Verbal creativity and schizotypal personality in relation toprefrontal hemispheric laterality: A behavioral and near-infrared optical imaging study", Schizophrenia Research, Vol. 80 No. 2-3, pp. 271-282. https://doi.org/10.1016/j.schres.2005.06.016

Gibson, C., Folley, B.S. and Park, S. (2009), "Enhanced divergent thinking and creativity in musicians: a behavioral and near-infrared spectroscopy study", Brain and Cognition, Vol. 69 No. 1, pp. 162-169. https://doi.org/10.1016/j.bandc.2008.07.009

Goel, V. (1995), Sketch of Thought, MIT Press, London.

Hoshi, Y., Huang, J., Kohri, S., Iguchi, Y., Naya, M. et al. (2009), "Recognition of human emotions from cerebral blood flow changes in the frontal region: A study with event-related near-infrared spectroscopy", Journal of Neuroimaging, Vol. 21 No. 2, pp. 94-101. https://doi.org/10.1111/j.1552-6569.2009.00454.x

Kato, T., Otagiri, S., Nagamori, Y. and Izu, Y. (2016), "Comparison of Hand and Computer Drawings Using Nearinfrared Spectroscopy", Proceedings of KEER 2016 6th International Kansei Engineering and Emotion Research Conference, Leeds, United Kingdom, August 31 - September 2.

Kowatari, Y., Lee, S.H., Yamamura, H., Nagamori, Y., Levy, P. et al. (2009), "Neural networks involved in artistic creativity", Human Brain Mapping, Vol. 30 No. 5, pp. 1678-1690. https://doi.org/10.1002/hbm.20633

Matsuoka, Y. (2000), Design Science, Maruzen, Tokyo.

Nagamori, Y., Nakajima, M., Yokoi, T. and Yamanaka, T. (2009), "Analysis of the brain activity at the chair design task with Lego bricks”, Journal of Japan Society of Kansei Engineering, Vol. 9 No. 1, pp. 51-60. https://doi.org/10.5057/jjske.J090209-1

Peña, M., Maki, A., Kovačić, D., Dehaene-Lambertz, G., Koizumi, H. et al. (2003), "Sounds and silence: An optical topography study of language recognition at birth", Proceedings of the National Academy of Sciences of the United States of America, Vol. 100 No. 20, pp. 11702-11705. https://doi.org/10.1073/pnas.1934290100

Plattner, H., Meinel, C. and Leifer, L. (2011), Design thinking: Understand-Improve-Apply, Springer-Verlag, Berlin.

Dr. Takeo Kato, Ph.D. in Engineering

Keio University, Mechanical Engineering

3-14-1 Hiyoshi, Kohoku-ku, 223-8522 Yokohama, Japan

Email: kato@mech.keio.ac.jp 